\title{
EDITORIAL
}

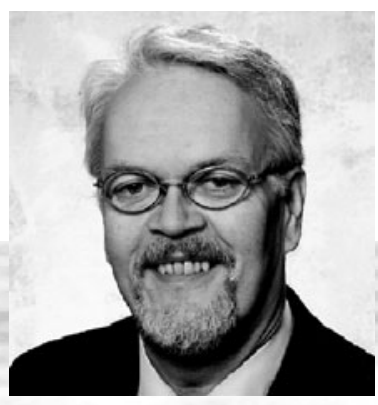

Hermann Engesser

Chefredakteur

\section{Reality broken?}

Warum eigentlich auf die Zukunft warten, wenn wir diese selbst machen können? Vor ein paar Jahren las man deshalb in einschlägigen Instituten Sciencefiction. Dieses Tool scheint aus der Mode gekommen zu sein. Erstes Mittel der Wahl sind heute Computerspiele. Das Abenteuer besteht im collaborativen Spiel und noch viel mehr im collaborativen Entwickeln solcher Spiele. Folgerichtig wurde auf der Siggraph 2012 für die Keynote Jane McGonigal eingeladen, Director of Game Research and Development am Institute for the Future in Palo Alto. Sie hat über Ihre Arbeit an der Zukunft den Bestseller Reality Is Broken: Why Games Make us Better and How they Can Change the World geschrieben. Dafür benötigt man natürlich leistungsstarke Rechner. Multicore selbstverständlich. Und immer wieder neue Erkenntnisse über die Wirklichkeit, die den Zukunftsarbeitern so viel Widerstand bietet. Man kann ja nun beispielsweise Haare, Oberflächen, Beleuchtung algorithmisieren und simulieren und doch ist da immer noch etwas mehr - in der Realität. Etwa die schnellen, unmerklichen Vibrationen und bewusst kaum wahrnehmbaren Bewegungen im menschlichen Gesicht, die dann einen wahrnehmbaren Unterschied zwischen einem realen Menschen und einer Animation ausmachen. Dieses Thema zog sich wie ein roter Faden durch die Siggraph und von dessen adäquater Lösung verspricht sich so mancher den Eintritt in das Uncanny Valley. Vielleicht meinte McGonigal dieses Ziel, wenn sie die Prognose wagt, dass Game Design in 20 Jahren nobelpreiswürdig sein wird.

Eine rechentechnisch viel mächtigere Installation als alle Siggraph-Rechner zusammengenommen ging offiziell am 20. Juli 2012 in Betrieb: SuperMUC, der neue Höchstleistungsrechner des Leibniz-Rechenzentrums der Bayerischen Akademie der Wissenschaften, das gleichzeitig den 50. Geburtstag feierte. SuperMUC ist mit mehr als 3 Petaflops Rechenleistung der viertschnellste Rechner der Welt und der schnellste Rechner Europas. Er verfügt über mehr als 155.000 Rechenkerne. Mehr als 330 Terabyte Hauptspeicher stehen für die zu verarbeitenden Daten zur Verfügung, die über ein nichtblockierendes InfiniBand-Netzwerk mit Fat Tree-Topologie kommuniziert werden können. Darüber hinaus können bis zu 10 Petabyte Daten in einem parallelen GPFS-Dateisystem zwischengespeichert werden. Weitere Informationen finden Sie im Forum.

Den Kontrapunkt konzipiert Gunter Dueck. Er macht sich auf die Suche nach gesundem Menschenverstand und stellt fest, dass die Regeln zu starr werden und alles in Ordnungen gepresst werden muss. Wo bleibt die Freiheit, etwas außerhalb der Prozesse zu entscheiden? Sind wir mittlerweile so weit, dass Ausnahmen als anrüchig gelten? Darf man gar nichts mehr selbst entscheiden? Auch nicht, wenn die Geschäftsprozesse Unsinn vorschreiben? Wo bleibt die Vernunft?

In einer Reihe von Beiträgen werden in diesem Heft verschiedenen Facetten der Informatik beleuchtet. Wir wünschen eine vergnügliche Fachlektüre.

\section{Hermann Engesser,}

\section{Chefredakteur Informatik-Spektrum}

